This is an Accepted Manuscript of an article published by Taylor \& Francis in Digital Creativity on 21 July 2017 , available online: http://www.tandfonline.com/10.1080/14626268.2017.1353524 


\title{
Water, image, gesture and sound: composing and performing an interactive audiovisual work
}

\section{Linda Walsh, Andrew Bluff \& Andrew Johnston}

Faculty of Engineering and Information Technology, Creativity and Cognition Studios, University of Technology Sydney, Australia

\begin{abstract}
Performing and composing for interactive audiovisual system presents many challenges to the performer. Working with visual, sonic and gestural components requires new skills and new ways of thinking about performance. However, there are few studies that focus on performer experience with interactive systems. We present the work Blue Space for oboe and interactive audiovisual system, highlighting the evolving process of the collaborative development of the work. We consider how musical and technical demands interact in this process, and outline the challenges of performing with interactive systems. Using the development of the work Blue Space as a self-reflective case study, we examine the role of gestures in interactive performance and identify new modes of performance.
\end{abstract}

\section{Keywords}

audiovisual, interactive system, gesture, visual music, sound and image

\section{Introduction}

This paper explores music performance with interactive audiovisual systems, focusing on the interactions between instrumental performers, collaborators and technical systems. An interactive system, according to Edmonds, is one in which human actions 'affect the behaviour of the system' (Candy and Edmonds 2011, p. 20). In practice, this means that the system adapts and responds to the human performer, who then in turn reacts to the system output, creating a fluid and dynamic performance environment. The complex interrelationships between the components, including live and processed audio, visuals, gestures and musical elements, pose many challenges for performers. This requires the development of new skills and approaches to performance, and a broadening of the scope of what may traditionally have been considered by musicians to be 'performance'. As performers and interaction designers adapt and develop new skills, new performance paradigms open areas of research in performer experience, interactivity and music making processes.

Many of the interactive audiovisual works created in this field are unique, custommade examples that are driven by the musical interests of particular individuals, combined with technology that is often adapted or repurposed for specific works. The field of practice is highly diverse, flexible, and constantly adopting, and adapting to, new technologies. This makes categorization and generalization difficult, and partly explains why terms such as 'audiovisual music', 'multimedia' and 'visual music' remain difficult to clearly define.

The works discussed in this paper are outcomes of practice-based research that 
aims to help document and understand creative practices in this changing field. The development of the new works for oboe and interactive system described here has been taking place over the past four years at the University of Technology Sydney. One of the objectives has been to develop works for oboe that take full advantage of the possibilities of technology to position the oboe in a contemporary performance context. We present a new work, Blue Space, for oboe and interactive audiovisual system, and discuss its musical and technical background and evolution from earlier works. Video of highlights from the Blue Space performance can be viewed at https://vimeo.com/157974674.

Following an approach drawing on Schön's concept of 'reflection on action' (Schön 1983) and Gruber's (1988) 'evolving systems approach' based on examining individuals closely, we focus on performer experience and creative practices during the evolution of new works over time. The aim is to examine the creative process in this particular context, and how diverse components in audiovisual works can be linked in a complex interactive environment

A survey of related works incorporating acoustic musical instruments, human performers and computer generated audiovisuals is presented. This is followed by a discussion of the three new works we have developed, and their evolution. The technical set up of our interactive system is explained, followed by a discussion of the challenges and opportunities of performance with our interactive audiovisual system from a performer's perspective.

\section{Background}

The interactive performance works that are the focus of this paper include acoustic musical instruments, human performers and computer generated sound and visuals. This section outlines related work with a range of approaches to the relationships between sound and image, providing context for the new work discussed later in the paper.

Jaroslaw Kapuscinski is a concert pianist and intermedia composer who creates works in which musical instruments are used to control multimedia content. He considers that sound and image are equal components, and uses the term intermedia to describe his work. He sees a connection in his work to Fluxus, because it sits conceptually between media (Kapuscinski and Sanchez 2010). Because of the intricate intertwining of the audio and visual layers, Kapuscinski suggests that these two aspects should be developed concurrently. He strongly recommends that a single artist should work on both the audio and visual components in order to achieve attention to detail and tight integration of the components (Kapuscinski 1997).

In Kapuscinski's work Counterlines (2010), a fundamental analogy was drawn between melodic intervals and lines. Using Max/MSP/Jitter and projection, the pianist generates graphic elements by playing. Meanwhile, the graphic performer's drawing gestures generate the sound of piano notes. A number of mapping strategies were tried whilst creating this work, revealing that direct linear mapping of parameters tended to be artistically limited, but was useful for analysis. In contrast, frequent changes of interface rules enabled more expressive freedom both to individual layers and the composition as a whole (Kapuscinski and Sanchez 2010). Establishing visible 
causality of who was controlling what appeared to contribute to 'performance believability' (Kapuscinski 1997). In addition to integrating sound and image through linking of layers in different ways, Kapuscinski suggests that inclusion of some pre-composed material contributed refinement and expressive precision in Counterlines (Sanchez and Kapuscinski 2010). Kapuscinski's background in classical music has enabled him to apply many characteristics of musical structure and traditional composition to the visual aspects of his work. His attention to clarity of structure, and specific techniques of linking of audio and visual components are successful partly because of his preference for simple components, which allow structures to be clearly revealed (Kapuscinski and Sanchez 2010).

Clarinetist Brigid Burke is another example of a performer/ composer who combines multiple media, improvisation, composition and live performance in her audiovisual work. Addressing the challenge of combining these diverse elements, Burke uses layering as a structural technique. Her 2014 work Escapee Gloss includes a wide range of components such as photos, videos, drawings and paintings alongside live and processed sound. Layering of both audio and visual components, allows her to quickly develop a wide range of materials that are transformed in many ways though their juxtaposition with other components (Burke Rigo 2014).

'During the performance, one is continually creating new musical ideas that trigger fresh sonic improvisations that then influence different combinations of visual layering that continually and fluidly change the balance between the visual and audio output. Both are reacting off each other at all times.
The visual output is based on an abstract representation of how the music is progressing. The audio is affected by the live video feed, by the lighting and by the amount the performers interact with the footage in real-time' (Burke Rigo 2014, p. 90).

While Burke's work demonstrates a twoway interaction between sound and visuals, Dannenberg's approach is to think of images as an 'integral part of the music', and not 'as an interpretation or accompaniment to audio'. By connecting images to an 'underlying musical deep structure' he claims that audiences may perceive emotional, expressive or abstract connections between sound and image (Dannenberg 2005). Sharing Kapuscinski's view on mapping, Dannenberg believes that if the connections between sound and image are too obvious, audience interest will not be sustained.

An important precedent to our work Blue Space is Golan Levin's Messa di Voce (2003), which uses a similar approach to sound recognition and motion detection. Messa di Voce uses a head tracking system on the vocalists and projects the visualizations so they 'appear to emerge directly from the performers' mouths. In some of the visualizations, projected graphical elements not only represent vocal sounds visually, but also serve as a playable interactive interface with which the sounds they depict can be retriggered and manipulated by the performers' (Levin and Lieberman 2004). In this respect, visuals are conceived as a controller in some sections of Messa di Voce, in a similar way to Blue Space, where the visuals are not only generated by the sound but are also a means of interacting with it.

Along these lines, Momeni and Henry (2006) propose the integration of sound 
and visuals using software models of physical objects. In their approach the models are used as a mediating layer between performer gesture and computer generated audiovisuals. The performer manipulates the models, which move in response in ways that are (usually) physically plausible. These movements are then used as the source of both sounds and visuals. Because sounds and visuals are grounded in the same physics based movements, the idea is that performers and audiences perceive an intrinsic connection between them, and are able to more intuitively understand how to interact with them. Henry has made use of this approach in several works including Egrégore (2011) and Morphist (2015). ${ }^{1}$ Our use of fluid simulations in Blue Space directly draws on this technique.

One of the significant research areas of interactive computer based music is the study of performer gestures. However, explicit consideration of the relationships between performer gestures and musical performance is by no means a new idea, as Bulwer's 1644 survey of gestures attests (Morrel-Samuels 1990). Hand gestures such as pointing were seen as a connection between the body of the performer and the mind. This recurring theme remains an important component of baroque vocal performance, and has resurfaced in digital instrument design, as pointed out by Garth Paine. He claims that 'the relationship of gesture to mind is critical in new interface design' because gesture is the first moment of excitation and engagement (Paine 2004).

Hand gestures were used in a new way with the 1920's theremin, controlling the volume and pitch of the instrument without physically touching it. 'The Hands' (Michael Waisvisz) premiered in 1984, Sonami's 'Lady's Glove', first developed in 1991, and Imogen Heap's 'The Gloves'
(2011) are further developments of the use of hand gesture as a controller.

Gestures trigger and manipulate sound and visuals in a unique blend of drumming and dance in Alon Ilsar's Airsticks. Ilsar argues that greater visual impact can be achieved by carefully considering the choreography of gestures. At times he will take a 'gesture first' approach to mapping, in which gestures are choreographed and then linked to sound, rather than the more usual 'sound first' approach where given sound or synthesis elements are mapped to gestures (Ilsar and Johnston 2015).

The natural bodily gestures of playing an acoustic instrument can also convey significant musical and structural information and contribute to audience appreciation of the music (Vines et al. 2004). Close attention to a string quartet in a live concert reveals the performers' immediate analysis, interpretation and reaction to other players' gestures. Extremely fine-grained adjustments in response to other players are a constant feature of live acoustic music. These can affect many musical components such as tone colour, dynamics, speed and intensity. In this respect, musical performance has always been interactive, long before computers were invented, and long before interactivity was a word. However, digitally capturing performer gestures and using the data to shape visuals and sound certainly opens new pathways for musical thinking and performing, including, as Paine suggests, 'the breaking of the excitationsonification bindings' (Paine 2004). This 'break' can be seen in works such as Tanaka's BioMuse (1992), which uses muscular and neural activity to create music, and James Maher's Laminar Flow (2015), using data from the flautist's vascular system. 
In order to digitise performer movement and gestures, diverse methods of motion capture have been explored. Options for instrument tracking include motion capture suits and gloves, video analysis, and for string instruments, bow motion and acceleration tracking. Video analysis is the preferred method for tracking of Jensenius and Johnson's (2012) electric violin. While acknowledging that video analysis is one of the slower and less precise techniques to track motion, they found it had the advantage of being unobtrusive to the performer. Their experiments suggest that violin bow tracking influences the performer behaviour when players know it is being used as a control parameter, and they also point out that bow movement may not be artistically very interesting (Jensenius and Johnson 2012). Violinist Mari Kimura's 2010 IRCAM residency produced refinements to the Augmented Violin bow tracking and led to new compositional approaches and ways of creating interactive performances (Kimura et al. 2012). Her earlier works utilised realtime sound analysis to gather performer information, which had the advantage of being less obvious to the audience (Kimura 2003).

This brief overview suggests that music making with interactive visual systems is very diverse and highly individual. While gestural interaction is an important theme, the absence of clearly defined genres encourages open exploration and creates opportunities for musicians to tailor works according to individual interests and available technology. This background sets the scene for our own work, which is discussed in the next section.

\section{Overview of Blue Space}

Blue Space is a work for oboe and interactive audiovisual system that was composed and performed as a collaboration between the first author (oboist) and second author (digital artist). The piece is a seventeenminute semi-improvised exploration of water in its many states and is inspired by Gaston Bachelard's philosophical and poetic text $W$ ater and Dreams: An essay on the imagination of matter (Bachelard 1942/1983). In a nutshell, Bachelard's fundamental theme is that immediate, lived experience of the elements is the key to the imagination and to understanding the self. Bachelard wrote about both the symbolic meanings as well as the physical and poetic powers of water, encompassing tranquility, power, life and death. His ideas are used in Blue Space as a source of inspiration and a theme which links the diverse entities of the piece. Blue Space depicts water in diverse moods and states, such as fluid, vapour, ice crystals and rain. It is the culmination of a series of performance works by author 1 which explore the connections between water, image, sound and text.

Blue Space uses an interactive fluid simulation system, described in technical detail below, to create an immersive performance environment in which visual images are projected onto a semitranslucent scrim in response to the sounds and movements of the oboist. The oboist is behind the scrim yet still visible behind the reactive images, forming an immersive melding of live performer with digital imagery. At the same time, the interactive system records and manipulates the oboe sound almost instantly, contributing an additional layer of sound to the live oboe.

The oboe movement is integrated into the piece using a custom made infrared light attached to the bell, which is tracked by the digital system using a high quality infrared point grey firewire camera and a two dimensional OpenCV blob tracking algorithm. The oboe sound is recorded with a microphone attached to the instrument, which is patched into the 
digital system. This records the sounds created by the oboist and injects these sounds into particles which are visualized so they appear to be emanating from the oboe itself. The digital system uses a dynamic fluid system algorithm to mediate the movement of these particles as they float effortlessly around the projected canvas. The system detects the movement of the performer as she moves about the stage and these physical movements are translated into forces in the virtual fluid simulation, pushing the floating particles around the screen.

The system settings have infinite possibilities for variation and change throughout the piece, with mappings between the oboe, sound and images constantly morphing and evolving. These mappings can link the work's components in many different ways, for example oboe volume can affect colour and size of the images, pitch can influence the system audio mediation characteristics, and the movement of the oboe itself can influence the appearance and location of the visual output. System settings control the viscosity, shape, colour and number of fluid particles. This results in a fluid and highly variable set of materials with which to develop musical works. The following sections describe the work Blue Space from the perspectives of the performance, composition and its evolution and development. Alongside this written paper, the reader is pointed to a video of the first performance of the work, presented at the University of Newcastle in September 2015. This is available on the 'vimeo' video sharing website. (https://vimeo.com/180401259)

Blue Space is structured in fourteen different scenes, each linked to a section of Bachelard's text that explores water experiences in different ways. Each scene is marked by differing musical material, performer gestures and individually customised settings of the interactive system. These features combine in different configurations, creating layers of live and processed sound, visualisations and interactions. The organisation of the fourteen scenes and overall structure of the piece mirrors the concept of the water cycle, in which water molecules circulate in varying states through a cycle from liquid to vapour, and return to earth as liquid. Although each scene is a self contained exploration of water, there is a suggested narrative of the movement around the water cycle. This forms a loose structure for the explorations of the properties and experiences of water as liquid, vapour, ice and precipitation. For rehearsal and identification purposes each of the fourteen scenes has a name, for example 'underwater', 'vapour', 'swimming' and 'iceforms'.

\subsection{Evolution of works for oboe and interactive audiovisual system}

Over the past four years, three new works for oboe and interactive system have been developed at the University of Technology Sydney. These works are outcomes of practice-based research that aims to contribute to the understanding of creative practice using a self-reflective approach. One of the goals is to develop new repertoire for oboe and digital technologies.

Three original works for oboe and interactive system have been produced: Sound Stream (2012, revised 2014), Airflow (2013) and Blue Space (2015). The following section traces the evolution of these works and their development, leading to the new work Blue Space. We outline the technical set up of the interactive system, and then 
focus on the process of development of Blue Space.

The interactive system used in the development of our pieces has its origins in the dance work Encoded, performed in 2012 by Stalker Theatre. ${ }^{2}$ This used a fluid simulation system based on MSAFluid by Mehmet Akten, ${ }^{3}$ which was further developed by Andrew Johnston (2013; 2015). In reflecting on this work, Johnston notes the developing understanding of the impact of these kinds of interactive systems on creative work and performer experiences. He points to the importance of achieving robust and reliable technical solutions that allow performers to focus on the 'craft' of interaction (Johnston 2015). Johnston's earlier work with interactive systems shows that successful systems need to be intuitively understandable but also rich and complex in their response (Johnston 2009).

The addition of sound to Andrew Johnston's original visual system opened up new opportunities for adapting the system to musical settings. This led to the experimental works for oboe, Sound Stream ${ }^{4}$ and Airflow ${ }^{5}$ in 2013 (Johnston and Walsh 2013). These early experimental pieces were short works that focused mainly on improvisations based on non-standard oboe sounds such as breath, articulation sounds, and key clicks. They were largely created as exploratory works to test and evaluate the possibilities of the interactive system as a medium for musical composition. As a result, these works were more a series of short vignettes rather than cohesive musical compositions.

One of the early changes to the projection was to change from using a solid screen to a 'scrim' (sheer theatre curtain). The scrim allows the performer to be visible to the audience sometimes, but also intentionally obscured at times by the emerging images.
This set up was devised to give the appearance of the projections being suspended in space and the performer emerging from the projected image. When the change was made from the solid screen to the scrim, the advantage was that the performer could stand behind the scrim and see the visuals appearing in front of them. This had a significant impact on the ways in which the performer could interact with the emerging visuals and develop gestures to drive and create the visual output. (see section 5.2 below). The scrim added visual depth to the performance and produced a slightly softer appearance than a solid screen, which suited the water themes explored in Blue Space. Figure 1 shows the performer behind the emerging images, which are projected from the front

Figure 1. Scene from Blue Space showing performer behind images.

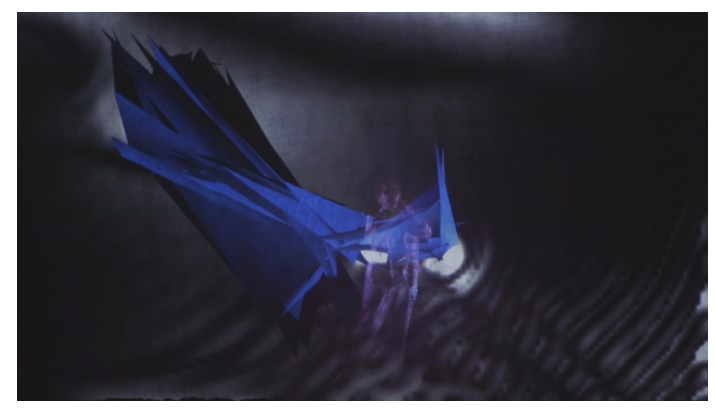

The earliest versions of the pieces used only white light projections, while in later versions richly coloured projections were used. Our experience was that too much colour could become overpowering, and detract from, rather than add to the scene. For this reason Blue Space uses a very constrained range of colour, predominantly shades of blue, green and white.

Looking back over the development of the pieces, each work could be seen as a model for the next, from which new ideas and avenues for exploration have emerged. The most recent work, Blue Space is a large-scale 
seventeen minute work with a fully developed musical structure and a strong philosophical basis, linked by an overarching theme of water. The first author's earliest audiovisual pieces were all based around ideas from water, stemming from a life long fascination with the correspondences between water and music. These include, for example, ideas of temporality, flow, patterns and periodicity. The potential of a fluid simulation system to explore this further had immediate and obvious appeal.

\subsection{Technical set-up}

The interactive system used in Blue Space is in essence a granular synthesizer and simple geometric visual renderer that is tightly coupled with a dynamic fluid system and motion capture. The hardware system remains similar to that used in the Airflow and Sound Stream works with a small instrument microphone used to capture the sound of the oboe, an infrared light and camera used to track the position of the oboe, and a laptop connected to projector and speakers to process and finally output the sound and vision in response to the human performer. As this was an evolution of the system that was developed for Encoded, Sound Stream and Airflow, it extends the custom written software components that were created in $\mathrm{C}++$, OpenFrameworks and PureData. Figure 2 shows the structure of the technical set up.

Figure 2. Technical diagramme for Blue Space.

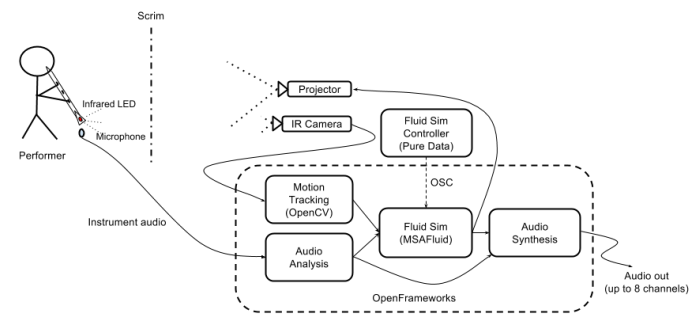

The PureData patch performs simple audio analysis of the incoming oboe stream, provides a complex screen-based user interface for controlling the audiovisual system and handles storing, retrieving and morphing of presets. As the screen-based user interface became more and more complex, dedicated and precise physical control over certain parameters like viscosity, volume, grain size and pitch were essential to provide more nuanced interaction with the live musical performer. To address this, the PureData patch was extended to allow control of these parameters via a physical controller, the Monome Arc 4.

The sound synthesis, visual rendering, motion capture calculations and physical simulations were all handled by OpenFrameworks software. While the dynamic fluid simulation system has remained largely untouched since its first use as a movement visualiser in the physical theatre performance, Encoded, the rest of the system has been significantly updated to work as an interactive audiovisual instrument capable of performing Blue Space. The motion capture system was altered to use blob-tracking so the system could detect the exact location of the oboe bell (with attached infra-red light) and therefore respond to sound from the oboe in such a way that the visuals appear to physically emit from the oboe itself. As the oboe makes sound, virtual particles are placed into the fluid simulation along with appropriate forces that are injected into the fluid to mimic the exertion of the performer. These particles float about the virtual fluid, which has simulated 'forces' applied to it by both acoustic sounds from the oboe and the gestural movement of the performer through the performance space.

The core of the visual rendering system revolves around drawing these particles in real-time using simple geometric 
techniques such as points, large dots, lines and solid polygons. In addition to this simple geometric rendering, post-render video effects such as motion trails, blur and glow were added to create rain and other water-like effects, albeit quite abstractly.

The addition of the granular sound engine is the most significant update from the Fluid's beginnings as a purely visual interaction system. This granular system was custom coded in $\mathrm{C}++$ and has evolved throughout the each of the oboe pieces Airflow, Sound Stream and Blue Space. The core idea of the granular system, like the geometric visual renderer, is that each virtual particle in the system is rendered as its own unique element that combines with other particles to provide the overall sonic picture. Each particle contains a small sample of audio (up to one second) that is recorded from the oboe at the time that the particle is created. This sampled audio is then constantly looped for as long as that particle is still in the system. The playback speed, loop size and volume of each individual recording (or sound grain) vary depending on the location, age and velocity of associated particle. The quantity and speed of particle movement combines with the input audio to create varying sonic effects that range from simple audio delays to garbled chatter-like response.

While the setup for the interactive fluid system is technically complex, the use of fluid simulation and tight integration of sound, gesture and vision is designed to create a very intuitive experience where every action of the performer creates unique but physically plausible audiovisual reactions in the system.

\section{Developing Blue Space}

The audiovisual composition was created collaboratively with both oboist and digital artist working together in the same space throughout the development period. Each individual artist was responsible for specific compositional elements. The oboist took care of musical composition, choreography and thematic content, while the digital artist was responsible for the visual aesthetics and sonic digital response. Each artist also contributed feedback and minor adjustments to the other's primary domain in rehearsal discussions. This codesign approach led to a synthesis of our respective skills and pushed us into areas beyond our individual expertise.

Weekly rehearsals of the piece were conducted from January to September 2015, culminating in a public performance at the University of Newcastle. Each rehearsal was around two hours long, and all were video recorded for study and reflection. The video recordings were an important tool for reflection on practice as they allowed a quasi third person view of the piece as it developed. Rehearsal videos were also useful for experimenting with the order of scenes, as the sequence of the video clips could easily be changed to give an impression of various configurations of the piece, saving a lot of time in rehearsal. The process of rehearsing can be categorised into three distinct stages. The first five rehearsals involved experimenting with different ways in which to visualise water. During this stage we developed about twenty-five individual system settings and performance ideas that depicted water in many different ways. These were various kinds of drops, flows, splashes, waves and ideas of freezing and melting. This first stage was primarily concerned with generating a pool of options for the piece. From these options, fourteen were chosen that we called 'scenes' which were used to build the final version of the piece.

The second stage over several months related to selecting and organising the scenes into order and ensuring that the 
ideas from Bachelard's text were integrated into the piece. This also involved developing the musical content played by the oboe, refining the visual design and working on ways in which the oboist's gestures could interact with the visuals.

The third stage of rehearsals focused on performance preparation. It revolved around ensuring that each scene transitioned smoothly to the next, and devising a set of visual and aural symbols so that the oboist and digital artist could communicate readiness to move to the next scene. This final stage also required technical refinements to ensure that the system itself was robust and reliable, and that it would be stable in performance.

In between the weekly rehearsals, each artist separately undertook further work on the piece at home. The oboist developed musical material, practiced using gestures and reviewed all the rehearsal videos, taking notes which formed a plan for the next rehearsal. The digital artist worked on the software, refining the interface for controlling the presets for each scene, adding visual effects such as blur, bloom and trail effects to enhance the visuals. In addition, he worked on ways to smoothly transition from one scene to the next which required fixing bugs and making the system reliable. We came to each rehearsal with new ideas to try and solutions to discuss and test.

As mentioned earlier in the paper, one of the significant layers in the work is its connection to Gaston Bachelard's philosophical text $W$ ater and Dreams: an essay on the imagination of matter, first published in 1942. An example of the direct influence of the philosophical text on the work can be seen in the exploration of Bachelard's idea of moulding and shaping clay. Mixing the elements of earth and water was viewed by Bachelard as a model for the imagination: 'I cannot emphasize too much how important the experience of fluidity and pliability is to an understanding of the psychology of the creative unconscious' (Bachelard 1942/1983, p. 13). In the scene we developed from these ideas, particles feature a high viscosity setting, so the particles resist flow and can form pliable semi-solid shapes. This allows the performer to create shapes on the screen, which agglomerate over time. Towards the end of the scene we reduce the viscosity of the fluid, analogous to adding more water to the clay to make it looser and more pliable, allowing the performer to stir the fluid more freely. At the same time, recorded sounds are replayed when the oboe is moved back through the initial recording location, creating a mixture of sounds as an analogy to the visual mixing. In this way, the visuals, the sound and Bachelard's moulding clay image are all interacting during a performance. Working with this idea led to the identification of mixing as a gestural mode, which is presented as one of the new modes of performance described later in this paper. Figure 3 shows the mixing gesture, viewed from behind the screen.

A further example, the 'swimming' scene, is based on Bachelard's extended description of swimming as an act of assertion over the power of nature. Bachelard likens swimming to other acts of domination of the sea such as seafaring and overcoming floods. He explains that the conquest with the elements is connected with overcoming fear and achieving confidence. In the 'swimming' scene, the oboist engages in a kind of combat with the images on the screen, which rise and fall like waves, and follow the oboist around in a menacing fashion. This is achieved by the digital artist, who controls where on the screen the projections appear, moving them around in response to the oboist throughout this scene. The oboist appears to push against the visual shapes, trying to 
escape them and overcome them. The system settings are such that the louder the oboe plays, the bigger the visual projections, so the combat is interactive. For the sound component of this scene, the idea of pushing and assertion is depicted by persistent repetition of small musical motifs based on pairs of notes. The rhythmic repetition mimics the idea of repeatedly pushing something away. As the oboe moves upward to its highest register, motif repetitions are louder and more assertive as if claiming dominance over the waves. (see figure 4)

Figure 3. Mixing gesture seen from behind the screen.
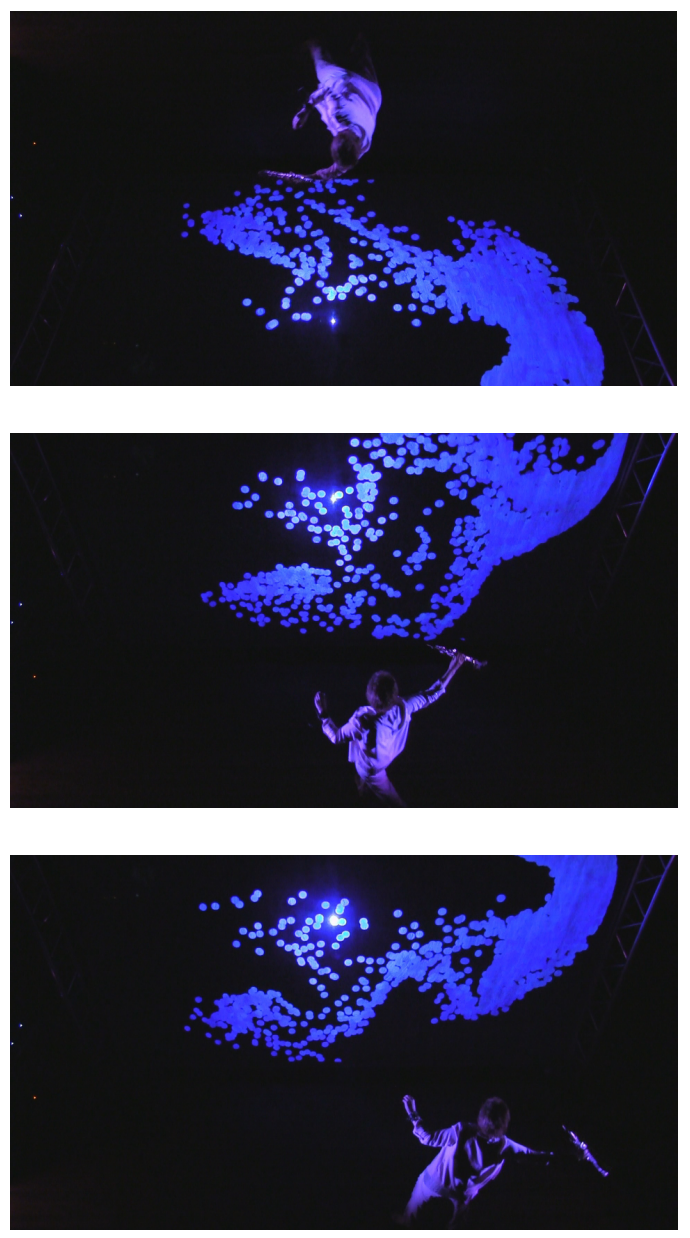

These descriptions take steps towards explaining how the abstract concepts of a complex literary text led towards concrete ideas that shape a musical performance.
Explanations of these thought processes demonstrate one of the ways in which the connections between water and music are explored in Blue Space.

Blue Space was conceived from the start with the conceptual framework of the water cycle in mind. While the piece was constructed with a series of semiimprovised 'scenes' as were the earlier pieces, in this work there was a much greater need for the individual scenes to meaningfully flow on to each other to form a conceptual progression through the piece. To achieve this flow, a considerable amount of work was involved in devising transitions from one scene to another. The system was always designed to morph between the complex set of values that define each individual scene, but sometimes considerable adjustments were needed to avoid unexpected system behaviour while morphing. In preparing for performances it was essential to solve these compositional and technical challenges and to ensure that transitions were musically convincing, reliable and stable. In this respect, the musical needs of the piece placed demands on the interactive system, which led to refinements and improvements of the technology.

Figure 4. Interacting

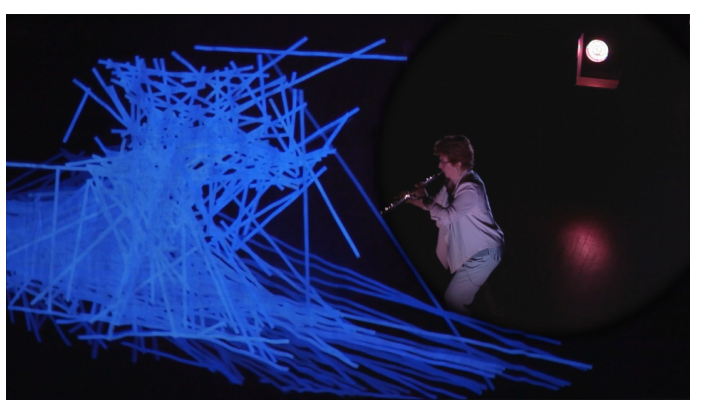




\subsection{Developing rehearsal techniques}

When preparing for a significant solo concert, an oboist would normally spend at least two to three hours per day practising. Working with an interactive system and a collaborating artist, this normal routine was not possible, as the system needed the resources of a specially equipped lab space, which could not be replicated at home. Weekly rehearsals were held in the lab, but without daily access to the bespoke system, the interaction with its specific sonic and visual outputs was difficult to prepare for.

In addition, as a largely improvised piece, the classical music practice of perfecting particular phrases, and solving technical challenges did not apply in the traditional way. The piece we were 'practising' in our weekly rehearsals did not exist yet, so a new kind of preparation was required. Rehearsal techniques had to be developed to suit the evolving piece. Performances did not require a perfectly controlled repetition of a score as traditional repertoire demands, but rather a more flexible approach that could spontaneously react to a wide range of visual and sonic system responses. Practising improvising with melodic motifs and rhythms helped to develop confidence when working together in rehearsals.

A practice system was devised using Ableton Live to approximate certain sounds of the granular synthesis treatment of the oboe in Blue Space. This became a kind of surrogate for practice, although obviously it could not react and adjust in the same way the system itself did. Exploring sounds and effects in Ableton Live provided opportunities to experiment with particular aspects of sound that could be applied later to the piece itself, such as how to react spontaneously to delay effects or changing pitch. Video recordings, which were made at every weekly rehearsal were a very useful practice tool, providing a quasi third-person view, used for reflecting on the work as it evolved. One advantage of using video in this way is that it did not disrupt the rehearsals or interfere with creative processes, which has been a recurring criticism of documenting artistic practice for the purpose of reflection and research.

Video was particularly useful for monitoring the development of performer gestures, one of the significant performance aspects of Blue Space. The use of gestures in the work evolved over time as we developed our understanding of how the oboist, digital artist and system could interact. Some of the gestures required a significant amount of movement around the space to influence the visuals as outlined below. However, large or sudden movements could compromise the oboe sound, by interfering with embouchure control or breath support. To avoid such compromises, developing particular gestures that minimised interfering with sound production, and considering the timing and pacing of those gestures became part of regular practice. When needing to move moving quickly from one side of the screen to the other, rapid small steps resulted in less embouchure disturbance than large steps, even though initially this was counter-intuitive. Practising at home while moving around, and pointing the oboe up and down as required in the piece, developed familiarity with controlling these movements while still playing.

\subsection{Developing a means for evaluation} Evaluating the piece as it progressed necessitated establishing criteria for making decisions throughout the process of composition. The piece grew from a cycle of reflective practice, involving experimentation, reflection, feedback, testing, and then repeating the process. Intuitive decisions were examined to reveal underlying tacit criteria, which became 
more explicit as the work progressed. In order to make aesthetic decisions, clarity of criteria was essential. These criteria were developed jointly between the oboist and digital artist as part of the process of composition. Judgements were initially based on quite general goals for the piece. It was only by close examination and discussion that more refined criteria could be clearly articulated. The criteria were that both the sound and visuals had clear connections with Bachelard's text, that the oboe sound was enhanced and not obliterated by the technology, and that the piece was executable in practice without system crashes. The visuals and sound feedback of the system were judged as suitable if they resulted in interesting reactive choreography from the oboist and evoked a feeling of water in an abstract sense. The general structure and detailed crafting were then continuously evaluated against these refined criteria to guide choices and establish whether improvements were needed.

Satisfying the criteria involved creating coherent connections between the sound, visuals and gestures of the piece. As each section of the piece was closely linked to a section of Bachelard's text, artistic decisions were guided by establishing strong analogical connections between the text and the diverse components of the piece. Developing the work with a constant refinement and reflection on the criteria allowed aesthetic decisions to be made clearly and confidently.

\section{Reflection on performance}

The interactive system used for Blue Space features almost immediate reaction of the system to performer gestures and sounds enabling a constantly changing performance environment to which performers must react in the moment.
Incorporating textual, visual, gestural and aural components simultaneously, demands rapid changes of performer focus in response to the system output. This involves a constantly changing balance between the sounds, visuals and the physical gestures used to interact with them.

Reflection on the performances of Blue Space indicates that the oboist does not always relate to the output of the system in the same way, but experiences a range of different modes of performance. These modes emerge in response to the complex and varied interactions of the components of the piece and the ability of the interactive system to provide a rich and varied environment for improvisation. The performance modes tend to overlap and combine with each other and usually more than one mode is in play at any given time. Within each scene it is common to 'flick' between modes to address multiple components of the work evolving in realtime, and to ensure the desired outcome is achieved. This finding is consistent with earlier research on composition and interaction with virtual musical instruments that demonstrates the use of multiple modes of interaction by composers and performers, and that several modes can operate concurrently (Brown 2003; Johnston 2009). Johnston's 2009 research on virtual musical instruments showed that performers move their focus between audio, visual and physical components. Their interactions with virtual instruments were classified as 'instrumental' when they wanted the instrument to respond consistently so they were able to fully control it, 'ornamental' when the system complemented or augmented their own sound and 'conversational' when engaging with the virtual instrument as if it were another performer. While Johnston studied performer behaviour for the purpose of improving the design of virtual instruments 
and understanding how musicians used them, in this paper we explore performer behaviour in the context of interacting with a system to develop a large scale musical composition. There are many intersections in these approaches, but here we distinguish between conceptual performance modes which are differentiated according to the predominant way the performer is thinking, and physical gestures which are specific performer movements devised to engage with the system. First, we outline the performance modes identified in Blue Space.

\subsection{Conceptual modes}

The performer experienced a number of different relationships when developing and performing the Blue Space work. These relationships can be categorized into four distinct modes depending on the specific focus towards sonic or visual and the actions of generating new material or interacting with existing material.

In sonic-compositional mode, the performer is mainly generating material, guided primarily by pre-planned musical ideas, such as making a particular sound, repeating a pattern, or using a particular harmony for musical purposes. This may include improvised sections within an overall predetermined structure. In this mode, the performer might not be thinking very much about the visual output of the system, and be happy to temporarily relinquish control of that aspect in order to focus on musical aspects.

In sonic-interactive mode, the performer focuses on the sound output of the system, and responds to it. The system can behave like a musical collaborator that replies, reacts and responds to the performer's input. Sounds of the oboe are recorded by the system and then manipulated using granular synthesis to create a vast range of sonic possibilities. The performer can interact spontaneously with this sonic output of the system, primarily in a conversational, improvised way.

In visual-compositional mode, the performer is guided primarily by preplanned visual ideas, aiming to generate particular visual colours or shapes on the screen. In some scenes, direct connections between the oboe sound and visual appearance allow the performer to manipulate the images, for example when the size or colour of the visuals is mapped to oboe volume.

In visual-interactive mode, the performer's focus is on interacting with the visual output of the system and influencing the appearance of the projections. The visualisations are primarily influenced by performer movements and gestures, but also by other aspects of playing such as volume and pitch.

It is important to note that these modes are not exclusive - it is often the case that the performer considers visual and sonic aspects of the work concurrently. Particularly in the visual modes, body gestures are a key part of generating and interacting with the visuals. The types of gestures we have identified are outlined in the following section.

\subsection{Gesture Categories}

Gestures are fully integrated into the performance language of Blue Space, contributing a physicality that draws attention to the human performer and helps to articulate and explore the themes of the work. The increasingly tight integration of gestures over the series of works we have developed is partly due to improved tracking of the oboe as well as a developing understanding of how gestures in their own right can be a feature of the work. Body movements and gestures are a natural part of playing a musical 
instrument, however in Blue Space, the gestures are more exaggerated and deliberately employed. Here, playing gestures are an important part of linking the concepts and ideas of the piece to the visuals. Gestures are the main means by which the performer can influence the visual output of the system made possible by tracking the oboe location using the infra-red light attached to the oboe bell. Because playing the oboe usually requires standing fairly still, working on these gestures and how to use them in the piece without compromising the production of sound on the oboe constituted an important aspect of the preparation for the piece. The gesture categories in Blue Space are differentiated according to the relationships between the performer and the system. In some situations, the gestures are aimed at controlling the visual output, while at other times the performer responds and interacts with the visual output without trying to control it. Many scenes feature several gestures types as the performer's relationship with the piece is constantly changing. Gestures include arm movements, pointing actions with the oboe, or moving the whole body up and down or right and left through the performance space.

Five main types of gestures have been identified:

1) enacting, in which the performer embodies a particular idea such as splashing water, or enacting the struggle against being underwater. This has some common elements with theatre, because the oboist intentionally acts out a role. Examples include the 'underwater' scene, which is an intentional theatrical portrayal or dramatization of struggling underwater, and the 'waterplay' scene, which portrays acting out a role of a child splashing in the water. In the enacting scenes, the oboist does not intentionally try to manipulate the visuals, but focuses on acting out an imagined scene. Although careful planning of the desired visual output and system settings has occurred in rehearsals, in the enacting scenes, the oboist allows the visuals to appear without trying to control them. In this sense, the enacting gestures can be viewed as a type of performance behaviour that can contain a number of differing physical gestural components, that all aim to depict imagined mental states.

2) painting, in which the performer actively 'paints' with the oboe's sounds and intentionally locates the visuals in particular areas of the screen, usually in response to where the projections are currently visible and where there is a suitable space on the screen. In this mode the oboe can draw shapes on the screen as shown in figure 4 . This is an intentional, conscious act in which the oboist follows the melodic contour with her gestures so that the oboe sound and the visuals work together to produce the impression that the oboe sound is visible.

3) mixing refers to a stirring action done with the outstretched arms holding the oboe without actually playing, which is used to create a blend of existing sounds. This works because the system can be set to record, manipulate and then replay sounds when the oboe returns to the location of the recording. At the same time, the images themselves are mixed and stirred by the gestures as indicated in figure 3. The mixing gesture capitalizes on a feature of the interactive system which associates recorded fragments of live sound with specific particles in the fluid simulation. Playback of these audio fragments occurs when oboe movements and sounds cause the particles to move. Thus, the mixing gesture stirs particles, causing them to move as if floating in fluid, while the audio associated with them plays back. The effect is to dynamically combine 
large numbers of sound fragments to create continually shifting timbres which are directly coupled to the movement of visual particles. Using mixing gestures, the oboist could twist and deform the images and trigger interesting playback outcomes, which we worked into the piece. In this case, this novel feature of the interactive system created the impetus for several sections of the piece. This was also an example of a way in which the system could expand the physical capacity of the oboist, both by producing several layers of sound simultaneously and by generating sound when the oboist was not actually blowing into the instrument.

4) interacting, in which the performer responds to the visualisations in a spontaneous way such as the scene depicting combat between waves and a swimmer as can be seen in figure 5 . In contrast to the enacting scenes, which derived their impetus from internal, emotional memories and ideas, the interacting gestures are responses to the sounds and visuals generated by the interactive system. In the 'swimming' scene, which depicts the assertion of the swimmer over the waves, the interaction is between the oboist and digital artist, via the interactive system. The digital artist sets the interactive system into a state where the visuals appear to be trying to dominate the performer. The oboist's movements around the space and arm gestures with the oboe respond directly to decisions and input from the digital artist. In a different scene, playful interaction between the live oboist and the rhythmic looping sonic output of the system is reminiscent of a conversation, which further shapes the oboe input. Decisions made in the rehearsal stages governed the parameters of the system settings to facilitate free interaction within the framework of the aims for the scene.
5) choreographing, in which gestures are guided by an overall visual design of the scene, considering where images need to be positioned to achieve a desired visual appearance, or to facilitate a particular type of motion. An example is seen in the 'descend' scene, where the oboe must start pointing up high, leaving space to descend lower, corresponding to the scene's purpose, which is to suggest descent into the depths of the ocean. With choreographing gestures, the performer is directly and intentionally controlling the location of the visuals. This occurs by using large body movements through the performance space, for example from one side of the screen to another, or up and down.

Reflection on these different types of interaction with the system indicates that a wide range of performer behaviours were used and the body had differing functions with differing levels of control over the visual output. Some of the gestures are augmented versions of the 'normal' instrumental gestures of playing the oboe. Others are new gestures that constitute a specific performance language for the work Blue Space which evolved during rehearsals. The oboe is used not only for producing sound, but has significant influence on the visual output of Blue Space. A short video demonstrating examples of the above categories of gestures can be viewed at: https://vimeo.com/194108748.

Figure 5. Painting.

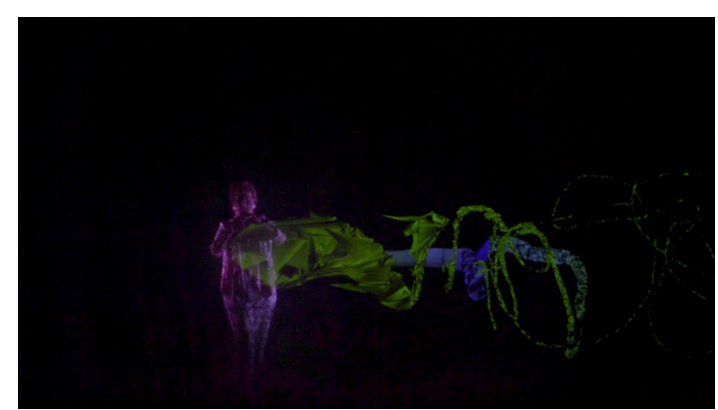




\subsection{Immersion and altered state experience}

An unexpected outcome of performing Blue Space was the oboist's experienceof an altered state of perception. We discuss this briefly here as an example of an outcome of the exploratory nature of practice-based research, which can reveal new research themes for future study. In three of the scenes, the oboist experienced a particular sense of actually 'being' in the scene: blowing ice crystals into space, floating in air, and making it rain. When the performer experienced this state, the body and the oboe temporarily disappeared from awareness, and a direct link was formed between imagination and visual output, creating a different experience of reality. It is interesting to note that this performer has never experienced an altered state before in an entire career of playing traditional repertoire. This altered state may be similar to Craig Vear's phenomenological experience of 'fluid immersion', resulting in 'thought-images which can be said to be a re-presentation of somewhere else and the creation of something else' (Vear 2014).

Albert (2012) suggests that there are two types of immersion, one that appears when there is a minimum of mediation and direct connection between the viewer and the work, causing 'devices to disappear'. The other form, as seen in Bill Viola's work Pneuma, prompts a self questioning that can emerge when objects and sounds are only partially suggested, causing the viewer to introspectively explore their own memories (Albert 2012). The altered reality state experienced in Blue Space may well be a combination of both these forms of immersion. While the system does respond directly and immediately to performer actions, the appearance of ice and rain, while easily identifiable, are not truly realistic representations of nature, so perhaps the imagination is triggered to supply the missing elements of realistic representation.

Closer scrutiny of when the altered state occurred in Blue Space reveals that in all cases the performer was in 'soniccompositional' mode playing only very simple or subtle musical material with almost no gestural input. At the same time, the system output was complex both sonically and visually. It may be that the rich system feedback enhanced the performer's awareness, leading to a heightened level of consciousness. Rokeby explains a similar phenomenon as an integration of the interactive system into our own proprioperceptive system such that 'the system and its responses are experienced in the same way that we experience our own body' (Rokeby 1998). Waterworth's concept of 'expanded embodiment' implies a 'change in the boundary between the self and the non-self (the other) that constitutes the world around one' (Waterworth and Waterworth 2014, p. 34).

Fels' (2000) studies of intimacy and embodiment in interactive artworks suggests four types of possible relationships between people and objects. Fels uses the term 'objects' to include systems, artworks and other people.

1. the person communicates with the object in a dialogue

2. the person embodies the object

3. the object communicates with the person

4. the object embodies the person. Fels suggests that the first two categories operate on a continuum rather than existing as separate entities. The degree to which a person embodies an object, Fels explains, is related to a measurement scale he calls 'intimacy' (Fels 2000). When intimacy is high, a person can communicate ideas and emotions through a device. The device becomes part of them as if an 
extension of the body, as in type two above. This may in part explain the experiences described above, of blowing the crystal shapes and rain directly onto the screen. However, the floating in air experience described above, is more similar to example four, as if the performer has been taken over by the system. Fels describes this as dissociation of the self, then viewing the self as being 'inside' the artwork. Describing this scenario of the object embodying the person in his interactive artwork the Iamascope, Fels explains:

'The imagery is just abstract enough with enough symmetry that the participant can look at the beautiful imagery separate from his own control... However, the image is the participant. Hence, the participant sees an abstraction of himself in the image and lets it manipulate him. The performer need only watch and listen as if from afar while the images seep through him' (Fels 2000).

Although Fels considers that the 'object embodying the person' is a further stage in a continuum from step two above, the experience of floating in air mentioned above was more immediate, and did not seem to be preceded by an experience of embodying the system. While a study of immersive states was not initially a goal of Blue Space, these performer experiences suggest that it is an area for potential further research, which we intend to study further.

\section{Conclusion}

This paper explored interactive audiovisual systems that use acoustic instruments, human performers and interactive computer systems. A survey of literature showed that while there are many individual approaches, the use of gesture in performance is a recurring theme. We outlined the evolution and development of Blue Space for oboe and interactive system which strongly features performer gestures. The new work demonstrates a new approach to oboe performance that blends elements of theatre, dance and improvisation. This created several challenges for the performer. Without existing precedents for this type of performance, new skills and refinements to existing skills were required. As described above, developing a rehearsal method to suit this type of performance was first needed to move the project forwards. Early in the process, a set of criteria to shape Blue Space had to be devised so that artistic decisions could be made. This required close interrogation of intuitive decisions and ongoing reflection on practice during the evolution of the piece.

Working with textual, visual, gestural and aural components simultaneously, demanded rapid switches of performer orientation in response to the system output. The use of gestures as a controller of the visuals required developing confidence with much larger than normal physical gestures while playing, necessitating careful planning so that playing was not compromised due to additional body movements. Reflection on these performer experiences resulted in the identification of the four new modes of performance and five categories of gesture, as outlined above.

An unexpected outcome of this work was the identification of the influence the interactive system on the performer's perception. If such a state could intentionally be induced, there may be interesting applications for the technique. Interactive audiovisual systems may be a useful vehicle for exploring these states of mind and seeking new knowledge about perception and consciousness in musical contexts. Further research is required to 
understand the particular circumstances in which a performer's perception can be influenced in this way.

The sustained engagement with the system over four years has pushed the technical and musical boundaries of both performers and system. This has demanded both refinements to the interactive system and

${ }^{1}$ http://www.chdh.net/

${ }^{2}$ Encoded: https://vimeo.com/55150853

${ }^{3}$ http://www.memo.tv/ofxmsafluid/

${ }^{4}$ Sound Stream:

https://vimeo.com/78448726 expanding the skillset of the performer to incorporate gestures. Like the early pieces we devised, our most recent work, Blue Space stands as a starting point for further developments.

${ }^{5}$ Airflow: https://vimeo.com/81951501

\section{Notes on contributors}

Linda Walsh is a Senior Lecturer at the University of Newcastle, Australia where she teaches oboe, improvisation and video skills. After postgraduate oboe study in Switzerland and France, Linda has appeared at contemporary music festivals in Berlin, Rome, Paris and Milan. In 2012 she was a guest artist at the Sonic Arts Research Centre in Belfast. Current interests include exploring new modes of expression for the oboe using computer sound processing and visuals in performance, which is the area of her $\mathrm{PhD}$ studies at the Creativity and Cognition Studios, University of Technology Sydney.

Andrew Bluff is a digital artist, software engineer and researcher at the University of Technology Sydney. Following his recent PhD studies combining immersive technology, interactive art and live performance, he is currently investigating new methods for mixed reality storytelling as a Postdoctoral Research Fellow at the UTS Animal Logic Academy. In addition to his collaborative performance works including Dot and the Kangaroo, Creature: Interactions and The Hour, Andrew has created music apps including DrumStudio and Mobile Phone Orchestra.

Andrew Johnston is a researcher, interaction/software designer and musician based in Sydney, Australia. His work focuses on the design of systems that support experimental, exploratory approaches to live performance, and the experiences and creative practices of the artists who use them. Andrew is codirector of the Creativity and Cognition Studios, an interdisciplinary research group working at the intersection of creativity and technology. He currently holds the position of Associate Professor in the School of Software, Faculty of Engineering and IT at the University of Technology Sydney. 


\section{References}

Albert, G. Immersion as category of audiovisual experience: From Long Beach to Hollywood. http://www5.unipv.it $/ \mathrm{wav} /$ index.php?option $=\mathrm{com}$ content\&view $=$ article\&id $=80 \&$ lang $=$ en .

Bachelard, G. 1942/1983. Ed. Stroud, JH. Water and Dreams: An essay on the imagination of matter. Dallas: The Dallas Institute of Humanities and Culture. (Orig. pub. L'Eau et les Rêves.)

Brown, A.R. 2003. Music Composition and the Computer: An examination of the work practices of five experienced composers. Ph.D, University of Queensland.

Burke Rigo, B. 2014. Escapee Gloss: A Symphony of Polymedia. PhD diss., University of Tasmania.

Candy, L. and E. Edmonds. 2011. Interacting: Art, research and the creative practitioner. United Kingdom: Libri Publishing.

Dannenberg, R.B. 2005. Interactive Visual Music: A Personal Perspective. Computer Music Journal 29, no 4: 25-35.

Fels, S. 2000. Intimacy and embodiment: implications for art and technology. In $A C M$ workshops on Multimedia, 13-16. Los Angeles: ACM.

Gruber, H.E. 1988. The evolving systems approach to creative work. Creativity Research Journal 1, no 1: 27-51.

Ilsar, A. and A. Johnston. 2015. Choreography in the Mapping of New Instruments. Paper presented at the ACM SIGCHI Conference on Creativity and Cognition, Glasgow.

Jensenius, A.R. and V. Johnson. 2012. Performing the electric violin in a sonic space. Computer Music Journal 36, no 4: 28-39.

Johnston, A. 2009. Interfaces for Musical Expression Based on Simulated Physical Models. $\mathrm{PhD} \mathrm{PhD}$, University of Technology, Sydney.

Johnston, A. 2013. Fluid Simulation as Full Body Audio-Visual Instrument. Paper presented at the International Conference on New Interfaces for Musical Expression, Korea.

Johnston, A. 2015. Conceptualising Interaction in Live Performance: Reflections on 'Encoded'. Paper presented at the 2nd International Workshop on Movement and Computing, Vancouver, Canada.

Johnston, A. and L. Walsh. 2013. Sound stream. In 9th ACM Conference on Creativity \& Cognition, 399-400. Sydney: ACM.

Kapuscinski, J. 1997. Mudras. Ph.D. PhD, University of California, San Diego.

Kapuscinski, J. and J. Sanchez. 2010. Counterlines: Studies in interfacing graphic and melodic lines.

Kimura, M. 2003. Creative process and performance practice of interactive computer music: A performer's tale. Organised Sound 8, no 3: 289-96.

Kimura, M., N. Rasamimanana, F. Bevilacqua, N. Schnell, B. Zamborlin and E. Fléty. 2012. Extracting human expression for interactive composition with the augmented violin. In International Conference on New Interfaces for Musical Expression, 99-102. Ann Arbor. 
Levin, G. and Z. Lieberman. 2004. In-situ speech visualization in real-time interactive installation and performance. Paper presented at the International Conference on Non-Photorealistic Animation and Rendering, Vancouver.

Momeni, A. and C. Henry. 2006. Dynamic Independent Mapping Layers for Concurrent Control of Audio and Video Synthesis. Computer Music Journal, 30, no 1: 49-66.

Morrel-Samuels, P. 1990. John Bulwer's 1644 treatise on gesture. Semiotica 79, no 3-4: 341-53.

Paine, G. 2004. Gesture and musical interaction: interactive engagement through dynamic morphology. Paper presented at the International Conference on New Interfaces for Musical Expression, Hamamatsu, Japan.

Rokeby, D. 1998. The construction of experience: Interface as content. In Digital Illusion: Entertaining the future with high technology, ed. Dodsworth, C, 27-48. Boston: AddisonWesley.

Sanchez, J. and J. Kapuscinski. 2010. Counterlines: a duet for piano and pen display. In Proceedings of the 28th international conference on buman factors in computing systems, 4765-70: ACM.

Schön, D.A. 1983. The reflective practitioner: How professionals think in action. New York: Basic Books.

Vear, C. 2014. Music, dimensions and play: composing for autonomous laptop musicians and improvising humans. Digital Creativity 25, no 4: 343-56.

Vines, B.W., M.M. Wanderley, C.L. Krumhansl, R.L. Nuzzo and D.J. Levitin. 2004. Performance gestures of musicians: What structural and emotional information do they convey? In Gesture-based communication in human-computer interaction, 468-78. Berlin: Springer.

Waterworth, J.A. and E.L. Waterworth. 2014. Altered, Expanded and Distributed Embodiment: the Three Stages of Interactive Presence. In Interacting with Presence: HCI and the Sense of Presence in Computer-mediated Environments, eds Riva, G, Waterworth, JA and Murray, D, 32. Warsaw/Berlin: De Gruyter Open. 